\title{
Kurze Mitteilungen
}

\section{Polyäthylen-beschichtetes Silicagel als Sorbens für organisch-chemische Fremdstoffe in Luft}

Polyethylene-Coated Silicagel as a Sorbent for Organic Pollutants in Air

Anreicherung von Pesticiden aus Luft; Sorption an polyäthylenbeschichtetem Silicagel

\section{F. Herzel und E. Lahmann}

Institut für Wasser-, Boden- und Lufthygiene des Bundesgesundheitsamtes, Berlin-Dahlem

Eingegangen am 21. Dezember 1972

Für die Bestimmung gasförmiger organischer Spurenstoffe in der Luft, wie z.B. Pflanzenschutzmittel, müssen diese Substanzen vor der eigentlichen Analyse angereichert werden. Eine Absorption in schwerflüchtigen organischen Lösungsmitteln (Waschflaschenprinzip) erwies sich aus verschiedenen Gründen als unzweckmäßig. Als „festes Lösungsmittel“ hat sich jedoch der Kunststoff Polyäthylen sehr gut bewährt. Die nachträgliche quantitative Elution der Substanzen aus dem Kunststoff ist jedoch wegen ihrer Diffusion in das Innere des Polyäthylensorbensteilchens problematisch. Diese Schwierigkeit kann umgangen werden, wenn lediglich eine dünne Schicht des Kunststoffs auf geeignete Träger aufgebracht wird.

Gute Absorptionsleistungen und eine quantitative Rückgewinnung von verschiedenartigen Pflanzenschutzmitteln konnten bei Luftproben erzielt werden, wenn Kieselgel $(0,2-0,5 \mathrm{~mm}$ Korngröße) mit etwa $10 \%$ seines Gewichtes mit Hart-Polyäthylen beschichtet wird. Hierzu wird das Polyäthylen in einem organischen Lösungsmittel (z.B. Decalin) gelöst und das Kieselgel eingetragen.

Es wurden Versuche mit zwei hintereinandergeschalteten Absorptionsröhrchen von $12 \mathrm{~mm} \varnothing$ durchgeführt, die in einer Höhe von 3 oder $6 \mathrm{~cm}$ mit dem polyäthylenbeschichteten Kieselgel ( $2 \mathrm{bzw} .4 \mathrm{~g}$ ) gefüllt waren und durch die ein Luftstrom mit geringen Pflanzenschutzmittelkonzentrationen in einer Menge von $2-3 \mathrm{~m}^{2}$ pro Tag gesaugt wurde.
Die Röhrchen mit dem Sorbens sind nach Elution mit 10 bis $20 \mathrm{ml}$ Aceton oder Benzol und anschließendem Trockensaugen sofort wieder einsatzbereit.

Die in jedem Falle um ein Vielfaches höheren Pflanzenschutzmittelmengen in dem ersten Röhrchen gegenüber dem nur für die Kontrolle der Absorption nachgeschalteten zweiten zeigte, daß der Rückhalteeffekt sehr gut ist. Das Verhältnis der ermittelten Mengen im Sorbens des ersten Röhrchens zu der im zweiten betrug bei den Versuchen von 250 bis 20000 : 1. Hierbei war eine Sättigung des Sorbens, d.h. das Auftreten nennenswerter Mengen im zweiten Röhrchen, noch keineswegs erreicht. Bei den in die Untersuchungen einbezogenen PflanzenschutzmittelWirkstoffen handelte es sich um die Insecticide Lindan, Aldrin, Dieldrin und DDT sowie um den Thiophosphorsäureester Parathion, weiterhin um chlorierte Biphenyle (Clophen A 60), sowie um die Herbicide Dichlobenil und Desmetryn.

Unbehandeltes Kieselgel zeigte zwar ebenfalls ein gutes Rückhaltevermögen für die getesteten Stoffe, besitzt jedoch gegenüber dem beschichteten Material einen höheren Strömungswiderstand und bewirkt bei empfindlichen Substanzen schon merkliche Zersetzungserscheinungen. Andere Sorbentien, wie Aluminiumoxide, zeigten diese nachteilige Eigenschaft in noch stärkerem Maße.

Höhere Luftdurchsätze für den Einsatz zu praktischen Außenluftuntersuchungen können durch Verwendung von Fritten-Einbaufiltern erreicht werden, die in senkrechter Lage einseitig mit dem kunststoffbeschichteten Kieselgel (ca. $12 \mathrm{~g}$ ) gefüllt werden. So können in $24 \mathrm{~h} 40 \mathrm{~m}^{3}$ Luft und mehr durchgesaugt werden. Die Resorptionsraten sind mit denen der Röhrchenversuche vergleichbar, d.h. der Rückhalteeffekt ist praktisch quantilativ. Das Sorbens wird hier zweckmäßigerweise in ein Chromatographierohr umgefüllt und dann mit etwa $25 \mathrm{ml}$ Aceton oder Benzol extrahiert.

Dr. F. Herzel

Institut für Wasser-, Boden- und Lufthygiene

des Bundesgesundheitsamtes

1000 Berlin 33, Corrensplatz 1

Deutschland 